\title{
Interobserver variations of target volume delineation and its impact on irradiated volume in accelerated partial breast irradiation with intraoperative interstitial breast implant
}

\author{
Ritu Raj Upreti, MSc, DipRPl, Ashwini Budrukkar, MD², Tabassum Wadasadawala, MD³, Shagun Misra, MD², \\ Lavanya Gurram, MD², Rima Pathak, MD², Deepak D. Deshpande, PhDl
}

'Department of Medical Physics, Tata Memorial Hospital, Parel, Mumbai, ${ }^{2}$ Department of Radiation Oncology, Tata Memorial Hospital, Parel, Mumbai, ${ }^{3}$ Department of Radiation Oncology, Advanced Centre for Treatment, Research and Education in Cancer, Kharghar, Navi Mumbai, India

\begin{abstract}
Purpose: To investigate the interobserver variations in delineation of lumpectomy cavity (LC) and clinical target volume (CTV), and its impact on irradiated volume in accelerated partial breast irradiation using intraoperative multicatheter brachytherapy.

Material and methods: Delineation of LC and CTV was done by five radiation oncologists on planning computed tomography (CT) scans of 20 patients with intraoperative interstitial breast implant. Cavity visualization index (CVI), four-point index ranging from $(0=$ poor $)$ to $(3=$ excellent $)$ was created and assigned by observers for each patient. In total, 200 contours for all observers and 100 treatment plans were evaluated. Spatial concordance (conformity index, $\mathrm{CI}_{\text {common' }}$, and $\left.\mathrm{CI}_{\mathrm{gen}}\right)$, average shift in the center of mass $(\mathrm{COM})$, and ratio of maximum and minimum volumes $\left(\mathrm{V}_{\max } /\right.$ $\mathrm{V}_{\min }$ ) of LC and CTV were quantified among all observers and statistically analyzed. Variation in active dwell positions $\left(0.5 \mathrm{~cm}\right.$ step) for each catheter, total reference air kerma (TRAK), volume enclosed by prescription isodose $\left(\mathrm{V}_{100 \%}\right)$ among observers and its spatial concordance were analyzed.

Results: The mean $\pm \mathrm{SD} \mathrm{CI}_{\text {common }}$ of LC and CTV was $0.54 \pm 0.09$, and $0.58 \pm 0.08$, respectively. Conformity index tends to increase, shift in COM and $\mathrm{V}_{\max } / \mathrm{V}_{\min }$ decrease significantly $(p<0.05)$, as CVI increased. Out of total 309 catheters, $29.8 \%$ catheters had no change, $29.8 \%$ and $17.5 \%$ catheters had variations of 1 and 2 dwell positions $(0.5 \mathrm{~cm}$ and $1 \mathrm{~cm})$, respectively. $9.3 \%$ catheters shown variations $\geq 10$ dwell positions $(5 \mathrm{~cm})$. The mean $\pm S D \mathrm{CI}_{\text {common }}$ of $\mathrm{V}_{100 \%}$ was $0.75 \pm 0.11$. The mean observed $\mathrm{V}_{\max } / \mathrm{V}_{\min }$ of prescription isodose and TRAK was 1.18 (range, 1.03 to 1.56) and 1.11 (range, 1.03 to 1.35), respectively.

Conclusions: Interobserver variability in delineation of target volume was found to be significantly related to CVI. Smaller variability was observed with excellent visualization of LC. Interobserver variations showed dosimetric impact on irradiation of breast tissue volume with prescription dose.

Key words: APBI, breast cancer, cavity visualization, interobserver variation.

\section{Purpose}

Accelerated partial breast irradiation (APBI) for early stage breast cancer using multi-catheter interstitial brachytherapy (MIB) has shown promising early results for selected subgroup of patients $[1,2,3,4,5,6,7,8,9]$. The APBI technique offers overall reduction of treatment time to 1 week, compared to 3-6 weeks of standard whole breast radiation therapy due to the irradiation of confined target volume around the lumpectomy cavity (LC).
Conventional dosimetry of MIB for APBI was carried out using a set of localization radiographs, which limited the three-dimensional (3D) definition of the target volume, thereby resulting in larger irradiated volume [10,11]. During the last decade, 3D image based brachytherapy has evolved, which provided clinically realistic dosimetric information on patient anatomy [10,11,12,13,14,15].

Active source positions in 3D brachytherapy are loaded in the vicinity of the 3D defined target volumes. Hence, 
the accurate delineation of the LC and clinical target volume (CTV) is essential to provide adequate dosimetric coverage and conformity. Variations in delineation of LC and CTV could result in the differences in treatment volumes, and may have impact on dosimetric as well as clinical outcome. Interobserver variability of tumor bed and target delineation for APBI using external beam radiotherapy has been extensively studied $[16,17,18,19,20]$; however, the literature available for MIB of breast implants is limited [21]. As per our knowledge, there is no study reporting the impact of interobserver variation in target delineation on irradiated volume.

Accelerated partial breast irradiation using MIB was initiated at our center in late 2000, and initially, 115 patients were treated by radiograph based planning [22]. Since 2005, we have adopted 3D brachytherapy, and have reported excellent local control rate in 140 women with long term follow-up [7]. The present study was carried out to investigate the interobserver variation amongst radiation oncologist in delineation of LC and CTV, and the impact of such variation on irradiated volume for APBI treatment using MIB in a busy tertiary cancer center.

\section{Material and methods}

\section{Patient selection and implant technique}

Twenty patients with early stage breast cancer treated with APBI using MIB were retrospectively included for the study. The details of patient selection, implant technique, and procedure have been published previously [11]. Patients with a tumor size up to $3 \mathrm{~cm}$, negative margins, and axillary lymph nodes were considered for intraoperative placement of the catheters in the LC (open cavity technique performed immediately after breast conserving surgery), which allowed a direct visualization of tumor bed. The tumor bed was marked with five radio-opaque clips placed at the superior, inferior, medial, and lateral borders as well as in the center of the posterior wall of the cavity.

\section{Imaging}

All patients underwent computed tomography (CT) scans with $0.3 \mathrm{~cm}$ slice thickness on Somatom Emotion (Siemens Medical Systems, Germany) and images were transferred to the brachytherapy treatment planning system (Nucletron, an Elekta company, Elekta AB, Stockholm, Sweden). Median time for surgery to CT imaging was 3 days.

\section{Cavity visualization}

We developed a cavity visualization index (CVI) to standardize the delineation process for open cavity technique practiced at our institution. The visualization of cavity was ranked using three parameters: clarity of cavity (includes visualization of seroma, air in cavity, and surgical clips), distinction of cavity with adjacent breast tissues, and distinction of cavity with muscle tissues. The four-point numeric index ranging from CVI $=0$ i.e. poor, all parameter unsatisfactory (poor visualization of cavity, which merges with adjacent tissues) to CVI $=3$ i.e. excellent, all parameters satisfactory (representing excellent visualization of cavity with clear distinction from breast and muscle tissues) was used in the current study (Figure 1).

Five radiation oncologists who specialize in the treatment of breast cancer participated as observers for this study. All observers independently assigned a CVI for each patient. The mode of the CVI was taken as CVI index for each patient.

\section{Delineation and treatment planning}

Each observer contoured the LC on the axial CT scans using most appropriate and consistent window level and width setting, and was blinded from reviewing the contours of the others. Clinical target volume was obtained by uniform volume expansion with $1 \mathrm{~cm}$ around the LC. Clinical target volume was edited by limiting the skin by $0.5 \mathrm{~cm}$ and up to the chest wall. For brachytherapy, CTV was considered as planning target volume (PTV).

To study the dosimetric impact of interobserver variation, treatment plans per patient for each observer were generated. Catheter reconstruction was carried out with $0.5 \mathrm{~cm}$ step size. The active loading length of each catheter was derived by auto margin of $0.5 \mathrm{~cm}$ over the CTV. The source positions were kept limited by $0.5 \mathrm{~cm}$ below the skin surface where the CTV was near the skin. Geometrical optimization on volume was carried out for treatment and dose were prescribed on $85 \%$ of basal dose points as in Paris system.

\section{Analysis}

The analysis was done by computing spatial concordance (conformity index, CI) of LC and CTV, which is ratio of common volume and encompassing volume drawn by all observers $\left(\mathrm{CI}_{\text {common }}\right)$. The generalized conformity index $\left(\mathrm{CI}_{\text {gen }}\right)$ was computed for LC and CTV.

The generalized conformity index $\left(\mathrm{CI}_{\text {gen }}\right)$ defined as the ratio of the sum of all overlapping volumes between pairs of observers and the sum of all overlapping and all non-overlapping volumes between the same pairs [23].

$$
\mathrm{CI}_{\text {gen }}=\frac{\Sigma_{\text {pairs ij }}|\mathrm{Ai} \cap \mathrm{Aj}|}{\Sigma_{\text {pairs ij }}|\mathrm{Ai} \cup \mathrm{Aj}|}
$$

Centre of mass (COM) was computed and mean distance for each pair wise comparison between COM of LC and CTV was quantified for all patients [16]. Ratio of maximum and minimum volumes among observers for each patient was computed.

To analyze the impact of interobserver variations of CTV in treatment planning, variation in active loading of source dwell positions for each tube for the CTV delineated by each observer (CTV1 to CTV5) was studied. For each plan, the volume enclosed by the prescription isodose $\left(\mathrm{V}_{100 \%}\right)$ and total reference air kerma (TRAK) was recorded. The spatial concordance $\left(\mathrm{CI}_{\text {common }}\right)$ of prescription isodose $\mathrm{V}_{100 \%}$ was computed for all observers. The ratio of maximum and minimum volumes of prescription isodose for each patient (Max/Min values of $\mathrm{V}_{100 \%}$ ) and Max/Min value of TRAK was also calculated. 

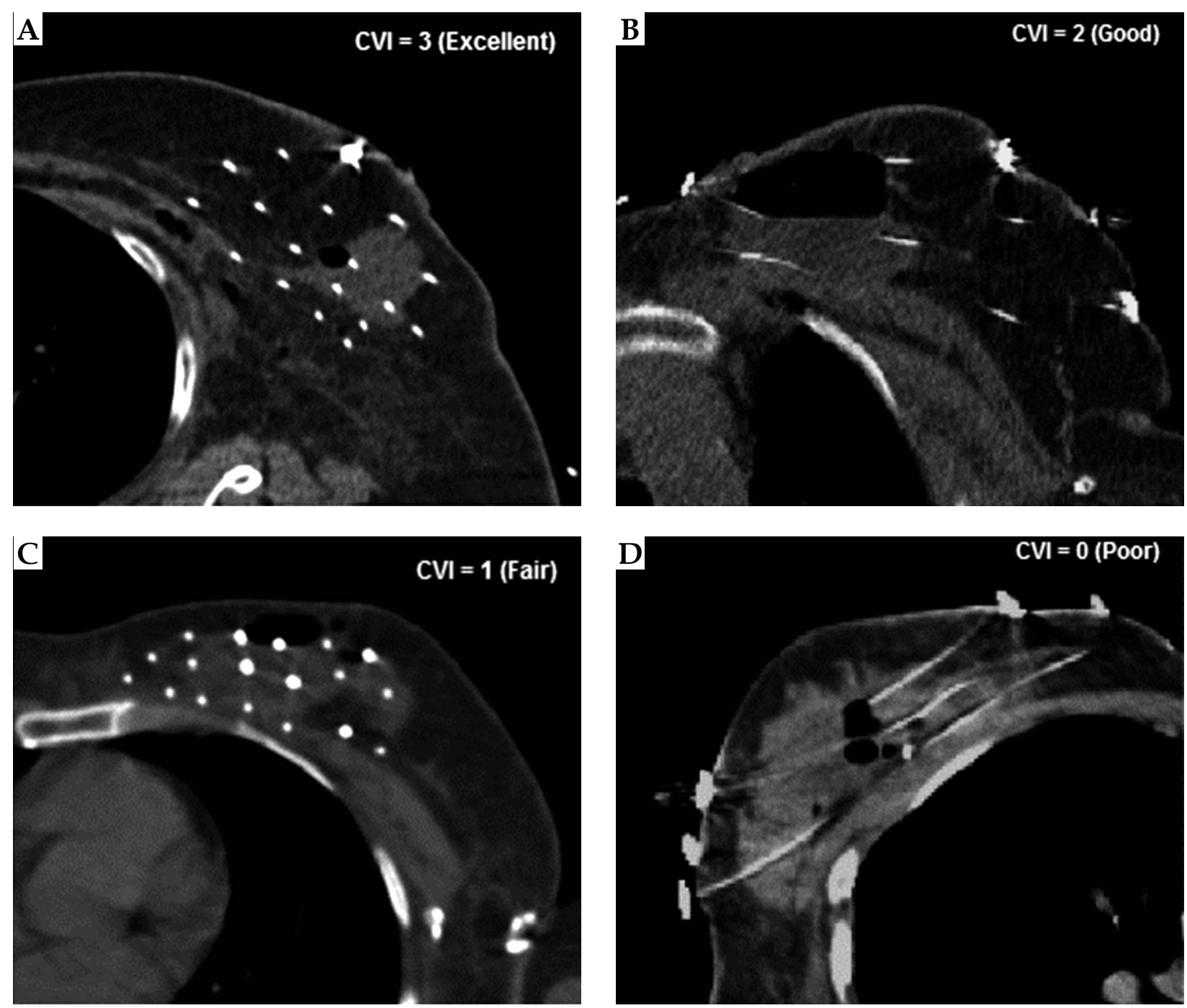

Fig. 1. The cavity visualization index. A) Cavity is well visualized with distinction from breast/muscle tissues. B) Cavity is visualized but not clearly distinguishable with either breast or muscle tissue. C) Cavity is visualized but not clearly distinguishable with both breast and muscle tissue. D) All parameters are not satisfactory

\section{Statistical analysis}

Statistical analysis was carried out using commercial Statistical Package for Social Sciences (SPSS version 20.0, IBM, Chicago). The coefficient of variation (COV) of volumes was computed. Wilcoxon signed-rank test was used to compare the volume of LC, CTV, and $\mathrm{V}_{100 \%}$ between each physician pair. One way ANOVA test was used to compare the measure of variability for LC and CTV with CVI levels.

\section{Results}

\section{Cavity visualization}

Out of 20 patients, four $(20 \%)$ patients had CVI $=1$, ten $(50 \%)$ patients had CVI $=2$, and six $(30 \%)$ patients had $\mathrm{CVI}=3$. There were no patients with $\mathrm{CVI}=0$.

\section{Contoured volumes}

Table 1 represents the pair wise comparison of CTV using Wilcoxon signed-rank test along with the median
Table 1. Comparison of clinical target volumes (CTV) among all observers

$\begin{array}{lll}\text { Pairwise } & \text { Median difference } & p \text { value } \\ \text { comparison } & \text { in CTV volume }\left(\mathrm{cm}^{3}\right)\end{array}$

\begin{tabular}{lcc}
\hline$\Delta_{21}$ (CTV2-CTV1) & 9.1 & 0.019 \\
\hline$\Delta_{31}$ (CTV3-CTV1) & 30.0 & 0.000 \\
\hline$\Delta_{41}$ (CTV4-CTV1) & 22.0 & 0.001 \\
\hline$\Delta_{15}$ (CTV1-CTV5) & 6.9 & 0.970 \\
\hline$\Delta_{32}$ (CTV3-CTV2) & 15.0 & 0.001 \\
\hline$\Delta_{42}$ (CTV4-CTV2) & 12.9 & 0.021 \\
\hline$\Delta_{25}$ (CTV2-CTV5) & 5.9 & 0.015 \\
\hline$\Delta_{34}$ (CTV3-CTV4) & 2.1 & 0.218 \\
\hline$\Delta_{35}$ (CTV3-CTV5) & 25.7 & 0.000 \\
\hline$\Delta_{45}$ (CTV4-CTV5) & 16.9 & 0.001
\end{tabular}

${ }^{*}$ For pairwise comparison data was arrange to keep median difference positive $\wedge$ Wilcoxon signed-rank test

CTV-clinical target volume, $\Delta$-difference in the volume 
difference in volumes for all observers. Significant difference was observed in the median volume of CTV between 8 pairs (out of 10 pairs in total) of observers. Summary of variability measures $\left(\mathrm{CI}, \mathrm{COM}\right.$ shift, and $\left.\mathrm{V}_{\max } / \mathrm{V}_{\min }\right)$ for volumes (LC, CTV) are given in Table 2. Median volume, COM shift, $\mathrm{CI}_{\text {common' }}$ and $\mathrm{CI}_{\text {gen }}$ for $\mathrm{LC}$ were $68 \mathrm{~cm}^{3}$, $0.26 \mathrm{~cm}, 0.56$ and 0.75 ; however, the values for CTV were $146.6 \mathrm{~cm}^{3}, 0.33 \mathrm{~cm}, 0.58$, and 0.78 , respectively. The mean $\mathrm{COV}( \pm \mathrm{SD}$ of mean) for LC was $0.11( \pm 0.06)$; however, it was $0.12( \pm 0.05)$ for CTV.

\section{Cavity visualization index and volumes}

Table 3 summarizes the variability of LC and CTV, and its relation to CVI. Significant decrease in COM shift of LC and CTV was observed with increase in CVI. Cases having fairy visible LC $(\mathrm{CVI}=1)$ showed large median COM shift of $0.46 \mathrm{~cm}$ for CTV, and $0.41 \mathrm{~cm}$ for LC. Cases with excellent visualization of cavity $(\mathrm{CVI}=3)$ had $\mathrm{COM}$ shift below $0.32 \mathrm{~cm}$ and $0.26 \mathrm{~cm}$ for CTV and LC, respectively. Significant increase of $\mathrm{CI}_{\text {common }}$ and $\mathrm{CI}_{\text {gen }}$ for $\mathrm{LC}$ and CTV was found with increase in CVI. For CVI $=3$, $\mathrm{CI}_{\text {common }}$ and $\mathrm{CI}_{\text {gen }}$ for CTV was 0.65 and 0.81 ; however, for $\mathrm{CVI}=1$, the respective values were 0.50 and 0.72 . The mean (SD) of $\mathrm{V}_{\max } / \mathrm{V}_{\min }$ was 1.32 (0.19) for $\mathrm{LC}$ and
$1.36(0.22)$ for CTV. Significant decrease in median of $\mathrm{V}_{\max } / \mathrm{V}_{\min }$ of LC $(p<0.029)$ and CTV $(p<0.008)$ volumes was found with increase in CVI. The Bonferroni post hoc analysis revealed the significant variation $(p<0.05)$ in all variability parameters for group of $\mathrm{CVI}=1$ and $\mathrm{CVI}=3$; however, all variability parameters for group of $\mathrm{CVI}=2$ and $\mathrm{CVI}=3$ was found insignificant. For group of $\mathrm{CVI}=1$ and $C V I=2$, variation in $\mathrm{C}_{\text {common }}, \mathrm{C}_{\text {gen }}$ of $\mathrm{LC}$ and $\mathrm{V}_{\max } /$ $\mathrm{V}_{\text {min }}$ of CTV was found significant $(p<0.05)$.

\section{Impact of interobserver variation on implant dosimetry}

Of the 20 patients, 3 patients $(15 \%)$ had two-plane, 14 patients $(70 \%)$ had three-plane, 2 patients $(10 \%)$ had four-plane, and one patient $(5 \%)$ had 5 plane implants. Average numbers of catheters used per patient were 11 (range, 10-13) in two-, 15 (range, 12-18) in three-, 20 (range, 16-25) in four-, and 22 in five-plane implants. A total of 309 implanted catheters (mean - 15.6, SD - 3.3) were evaluated to quantify the variations in source dwell positions from the median source loading due to interobserver variation in CTV and summarized in Table 4. Although volumetric variation of CTV was observed in all three dimensions among all observers, in major-

Table 2. Lumpectomy cavity and clinical target volume variability

\begin{tabular}{lccccccc} 
& \multicolumn{3}{c}{ Lumpectomy cavity $(\mathrm{LC})$} & \multicolumn{3}{c}{ Clinical target volume (CTV) } \\
\cline { 2 - 8 } & Mean & Median & SD & Mean & Median & SD \\
\hline Volume $\left(\mathrm{cm}^{3}\right)$ & 75.8 & 68.0 & 30.9 & 159.8 & 146.6 & 58.8 \\
\hline $\mathrm{Cl}_{\text {common }}$ & 0.54 & 0.56 & 0.09 & 0.58 & 0.58 & 0.08 \\
\hline $\mathrm{Cl}$ gen & 0.74 & 0.75 & 0.06 & 0.76 & 0.78 & 0.05 \\
\hline $\mathrm{COM}$ shift $(\mathrm{cm})$ & 0.27 & 0.26 & 0.10 & 0.34 & 0.33 & 0.13 \\
\hline $\mathrm{V}_{\text {max }} / \mathrm{V}_{\text {min }}$ & 1.32 & 1.26 & 0.19 & 1.36 & 1.34 & 0.22
\end{tabular}

$S D$ - standard deviation, $\mathrm{Cl}_{\text {common }}$ - conformity index among all observers, $\mathrm{Cl}_{\text {gen }}$ - generalized conformity index for all observers, COM shift - shift in the center of mass of the volume, $V_{\max }$ - maximum volume among observers, $V_{\text {min }}-$ minimum volume among observers

Table 3. Cavity visualization index (CVI) and variability of lumpectomy cavity and clinical target volumes with median value and range

\begin{tabular}{|c|c|c|c|c|}
\hline CVI & 1 & 2 & 3 & $\rho$ value* \\
\hline N & 4 & 10 & 6 & \\
\hline COM shift of LC (cm) & $0.41(0.29-0.46)$ & $0.26(0.19-0.52)$ & $0.18(0.12-0.26)$ & 0.004 \\
\hline COM shift of CTV $(\mathrm{cm})$ & $0.46(0.37-0.62)$ & $0.35(0.2-0.61)$ & $0.26(0.17-0.32)$ & 0.019 \\
\hline $\mathrm{Cl}_{\text {common }} \mathrm{LC}$ & $0.48(0.33-0.49)$ & $0.56(0.40-0.66)$ & $0.61(0.53-0.65)$ & 0.01 \\
\hline $\mathrm{Cl}_{\text {common }} \mathrm{CTV}$ & $0.50(0.39-0.56)$ & $0.59(0.45-0.70)$ & $0.65(0.51-0.68)$ & 0.02 \\
\hline $\mathrm{Cl}_{\text {gen }} \mathrm{LC}$ & $0.69(0.59-0.69)$ & $0.75(0.64-0.81)$ & $0.78(0.74-0.82)$ & 0.004 \\
\hline $\mathrm{Cl}_{\text {gen }} \mathrm{CTV}$ & $0.72(0.65-0.75)$ & $0.78(0.69-0.83)$ & $0.81(0.73-0.83)$ & 0.018 \\
\hline $\mathrm{V}_{\max } / \mathrm{V}_{\min }(\mathrm{LC})$ & $1.42(1.36-1.75)$ & $1.26(1.10-1.73)$ & $1.19(1.07-1.29)$ & 0.029 \\
\hline $\mathrm{V}_{\max } / \mathrm{V}_{\min }(\mathrm{CTV})$ & $1.57(1.44-2.02)$ & $1.29(1.14-1.68)$ & $1.20(1.15-1.50)$ & 0.008 \\
\hline
\end{tabular}

*One-way ANOVA test

LC - lumpectomy cavity, CTV - clinical target volume, $C I_{\text {common }}$ - conformity index among all observers, $C I_{\text {gen }}$ - generalized conformity index for all observers, $V_{\text {max }}-$ maximum volume among observers, $V_{\text {min }}$ - minimum volume among observers 
ity of catheters $(64.2 \%)$, variation in the source loading was observed along the catheters. Absence of all source active positions in the plan of any one of the observers was found in 18 catheters $(5.8 \%)$. This was due to the absence of CTV contour of the observer on those catheters lying at the peripheral location of CTV in 10 patients. In $29.8 \%$ of catheters, there was no change in the dwell positions, $29.8 \%$ of catheters had variation of $1 \mathrm{dwell}$ position $(0.5 \mathrm{~cm})$, and $17.5 \%$ of catheters had variation of 2 dwell positions $(1 \mathrm{~cm})$. However, $9.3 \%$ of catheters showed variations $\geq 10$ dwell positions $(5 \mathrm{~cm})$. Table 5 represents the impact of interobserver variation on dosimetric parameters. Median volume of prescription isodose $\mathrm{V}_{100 \%}$ was $134.2 \mathrm{~cm}^{3}$ (SD - $43.7 \mathrm{~cm}^{3}$; range, 74.4-229.6 $\mathrm{cm}^{3}$ ). The mean observed Max/Min value of $\mathrm{V}_{100 \%}$ was 1.18 (range, 1.03 to 1.56); however, its variation with CVI was found insignificant. The mean observed Max/Min value of TRAK was 1.11 (range, 1.03 to 1.35).

\section{Discussion}

Target delineation in 3D MIB for APBI is critical as it involves irradiation of partial breast volume alone, which is at higher risk of tumor relapse if not treated adequately. Moreover, uncertainty in the whole treatment procedure is contributed by target volume delineation, which is substantiated by geometric uncertainties in external beam radiotherapy, but not so in brachytherapy. This uncertainty is taken care by PTV margins in external radiotherapy, but in brachytherapy, the concept of PTV is not used. Errors are less forgiving and more severe in brachytherapy as sources lie within the target region, which if not defined precisely, can lead to reversal of the therapeutic ratio. Variation in CTV volumes had an impact on implant dosimetry, which resulted in different treatment volumes among the five observers in the current study. Literature search revealed many studies on interobserver variations $[16,17,18,19,20]$. However, limited work has been published for MIB for APBI using open cavity surgery [21].

Quantification of visualization of cavity was developed and used in many published studies $[17,24]$. The scale/score of cavity visualization in other published studies had 5 or 6 steps of scoring, mostly applicable for postoperative cavities. The median time for CT imaging after lumpectomy and intraoperative catheter placement for our patients was 3 days; therefore, cavity was always present in all of our cases. We have developed our own
Table 4. Impact of interobserver variation on active source positions

\begin{tabular}{lccc}
$\begin{array}{l}\text { Variations } \\
\text { in dwell } \\
\text { positions }\end{array}$ & $\begin{array}{c}\text { Variation in } \\
\mathrm{cm}\end{array}$ & $\begin{array}{c}\text { No of } \\
\text { catheters }\end{array}$ & $\begin{array}{c}\text { \% of total } \\
\text { catheters }\end{array}$ \\
\hline 0 & 0 & 92 & 29.8 \\
\hline 1 & 0.5 & 92 & 29.8 \\
\hline 2 & 1.0 & 54 & 17.5 \\
\hline 3 & 1.5 & 24 & 7.8 \\
\hline 4 & 2.0 & 14 & 4.5 \\
\hline 5 & 2.5 & 4 & 1.3 \\
\hline 10 & 5.0 & 21 & 6.8 \\
\hline 15 & 7.5 & 8 & 2.5 \\
\hline
\end{tabular}

cavity visualization index (CVI) for the intraoperative brachytherapy technique practiced at our Institute. Cavity visualization index, which is used in the present work, had 4 steps of scoring. The excellent visualization of cavity for our system has CVI $=3$ in contrast to the other system $[17,24]$ where Score ' 5 ' represent the best visualization.

Present study demonstrated the correlation of CVI with variability of delineation, better visualization resulted in better concordance, and decreased COM shift. For $\mathrm{CTV}$, median spatial concordance $\left(\mathrm{CI}_{\text {gen }}\right)$ was improved from 0.72 to 0.81 , and median COM displacement of CTV was decreased from $0.46 \mathrm{~cm}$ to $0.26 \mathrm{~cm}$, as the CVI increases from ' 1 ' to ' 3 '. Similar findings were reported by Landis et al. [16] for PTV; their values of $\mathrm{CI}_{\text {pair }}$ had improved from 0.57 to 0.87 and median COM were decreased from $0.69 \mathrm{~cm}$ to $0.15 \mathrm{~cm}$ with increase in visualization score.

The only published study of interobserver variation of APBI using multicatheter brachytherapy was by Major et al. [21]. The observed spatial concordance $\left(\mathrm{CI}_{\text {common }}\right.$ and $\left.\mathrm{CI}_{\text {gen }}\right)$ of cavity in post implant $\mathrm{CT}$ using guidelines was 0.36 and 0.56 , and the respective values of CTV were 0.54 and 0.70 . Our data showed better concordance on post implant planning $\mathrm{CT}$ images with values of $\mathrm{CI}_{\text {common' }}$ $\mathrm{CI}_{\text {gen }}$ for cavity and CTV was $0.54,0.74$, and $0.58,0.76$, respectively. This could be due to the differences in the

Table 5. Impact of interobserver variation on dosimetric parameters

\begin{tabular}{llllll} 
& Min & Max & Median & Mean & SD \\
\hline $\mathrm{V}_{100 \%}\left(\mathrm{~cm}^{3}\right)$ & 74.4 & 229.6 & 134.2 & 135.1 & 43.7 \\
\hline $\mathrm{Cl}_{\text {common }}$ of $\mathrm{V}_{100 \%}$ & 0.52 & 0.90 & 0.76 & 0.75 & 0.11 \\
\hline Max/Min of $\mathrm{V}_{100 \%}$ & 1.03 & 1.56 & 1.15 & 1.18 & 0.13 \\
\hline TRAK (cGy at $1 \mathrm{~m})$ & 0.18 & 0.32 & 0.24 & 0.24 & 0.04 \\
\hline Max/Min of TRAK & 1.03 & 1.35 & 1.09 & 1.11 & 0.07
\end{tabular}

$V_{100 \%}$ - volume of breast in $\mathrm{cm}^{3}$ receiving prescription isodose, Max/Min of $V_{100 \%}$ - ratio of maximum and minimum volume of breast volume receiving prescription isodose among all observers, TRAK - total reference air kerma in cGy at 1 meter 
timing and technique of implant as well as imaging protocol of the two institutes. In the Budapest series, catheters were not implanted immediately after lumpectomy; pre-implant CT imaging was done in all cases and catheters were inserted with the help of a template. However, at our institute, the intraoperative placement of the catheters was done immediately after lumpectomy as a single procedure without any pre-planning imaging. Free hand technique was utilized due to direct visualization of the tumor bed.

Struikmans et al. [20] had observed mean CI of 0.56 for boost CTV for external beam radiotherapy and they had concluded that each institute should determine their interobserver variability with respect to target volume. Reported CI of seroma volume for three observers by Petersen et al. [17] was 0.61. Similar to our observation, they had reported significant correlation between seroma clarity and conformity of seroma volumes.

Mean $\mathrm{V}_{\max } / \mathrm{V}_{\text {min }}$ of LC and CTV in our study was 1.32 and 1.36, respectively. Major et al. [21] have demonstrated that with contouring guidelines, the mean $\mathrm{V}_{\max } / \mathrm{V}_{\text {min }}$ ratio of CTV was decreased from 2.2 to 1.2 for oncologists having experience in open cavity surgery. However, it was 2.8 for observers without open cavity experience in absence of guidelines.

Recently GEC ESTRO (Groupe Européen de Curiethérapie European Society for Radiotherapy and Oncology) breast cancer working group (II) has published recommendations for the target delineation for accelerated or boost partial breast irradiation [25]. They have recommended the total safety margins of $2 \mathrm{~cm}$ for CTV in all 6 directions including surgical resection margins around the tumor. It was also recommended to have safety margin for CTV of at least $0.5 \mathrm{~cm}$ whenever surgical margins are larger than $2 \mathrm{~cm}$. Nevertheless, they were unclear whether recommended safety margin for CTV are sufficient to account for interobserver and intraobserver contouring variation. At our center, we used $1 \mathrm{~cm}$ margin from the cavity for CTV in majority of the patients. This essentially is due to wider surgical margins as can be seen from the low margin positive rates reported earlier [7]. GEC ESTRO also recommended the method to determine the boundaries of lumpectomy cavity utilizing seroma and surgical clips. For present study, the visualization of seroma, air in cavity, and surgical clips were used to define the clarity of cavity for CVI index.

In our work, we also considered the dosimetric impact of interobserver variation in target volume delineation. Active source dwell positions from all treatment plans were compared. Although $29.8 \%$ of catheters had shown a variation of only one dwell position from median active source position, catheters which were lying parallel or oblique and at the peripheral locations of the CTV showed larger variations in the active source positions. The variation of $\geq 10$ dwell positions $(5 \mathrm{~cm})$ were observed in 29/306 catheters, out of which 18/306 catheters (in 10 patients) were lying at the peripheral location of CTV showed variations in all active source positions. This could be due to absence of CTV contour along the catheters by any one of the observer. To avoid any subjectivity in dosimetric comparison, all plans were geomet- rical optimized on volume. Although variation of Max/ Min value of $\mathrm{V}_{100 \%}$ with CVS was not significant, the volume of prescription isodose $\left(\mathrm{V}_{100 \%}\right)$ had shown maximum variation of up to $56 \%$ with mean variation of $18 \%$.

Another way of reducing the dosimetric uncertainties is to have standardized institutional protocols for image acquisition and target volume delineation. Critical evaluation, review by peers, and constant training help to reduce further the interobserver variations. The limitation of our work is that we have not investigated the role of other imaging modalities (ultrasonography, magnetic resonance imaging etc.) in addition to CT for improvement in delineation of LC, as we had carried out the study retrospectively. Major et al. [21] have discussed and reviewed the role of other imaging modalities for delineating the tumor cavity, wherein the authors conclude that there is a need of further investigation in this area.

\section{Conclusions}

Significant interobserver variation in delineation of LC and CTV was observed for intraoperative multicatheter partial breast brachytherapy. Interobserver variability was found to be significantly related to CVI. Cases, in which the visualization of LC was excellent, demonstrated smaller variability in the delineation of LC and CTV. Interobserver variations showed an impact on the source positions along catheters, and thus have dosimetric impact on the volume of irradiated breast tissue with the prescription dose.

\section{Disclosure}

Authors report no conflict of interest.

\section{References}

1. Polgár C, Strnad V, Major T. Brachytherapy for partial breast irradiation: the European experience. Semin Radiat Oncol 2005; 15: 116-123.

2. Vicini FA, Arthur DW. Breast brachytherapy: North American experience. Semin Radiat Oncol 2005; 15: 108-115.

3. Kuske R, Winter K, W Douglas et al. Phase II trial of brachytherapy alone after lumpectomy for select breast cancer: toxicity analysis of RTOG 95-17. Int J Radiat Oncol Biol Phys 2006; 65: 45-51.

4. Ott OJ, Hildebrandt G, Pötter R et al. Accelerated partial breast irradiation with multi- catheter brachytherapy: local control, side effects and cosmetic outcome for 274 patients. Results of the German-Austrian multi-centre trial. Radiother Oncol 2007; 82: 281-286.

5. Yeo S, Kim J, Kwak G et al. Accelerated partial breast irradiation using multicatheter brachytherapy for select early-stage breast cancer: local control and toxicity. Radiat Oncol 2010; 5: 56.

6. Polgár C, Major T, Fodor J et al. Accelerated partial-breast irradiation using high-dose-rate interstitial brachytherapy: 12-year update of a prospective clinical study. Radiother Oncol 2010; 94: 274-289.

7. Budrukkar A, Lavanya G, Upreti R et al. Clinical outcomes of prospectively treated 140 women with early stage breast cancer using accelerated partial breast irradiation with 3 dimensional computerized tomography based brachytherapy. Radiother Oncol 2015; 115: 349-354. 
8. Strnad V, Ott OJ, Hildebrandt G et al. 5-year results of accelerated partial breast irradiation using sole interstitial multicatheter brachytherapy versus whole-breast irradiation with boost after breast-conserving surgery for low-risk invasive and in-situ carcinoma of the female breast: a randomised, phase 3, non-inferiority trial. Lancet 2016; 387: 229-238.

9. Bitter SM, Heffron-Cartwright $P$, Wennerstrom $C$ et al. WBRT vs. APBI: an interim report of patient satisfaction and outcomes. J Contemp Brachytherapy 2016; 8: 17-22.

10. Major T, Fodor J, Takácsi-Nagy Z et al. Evaluation of HDR interstitial breast implants planned by conventional and optimized CT-based dosimetry systems with respect to dose homogeneity and conformality. Strahlenther Onkol 2005; 181: 89-96.

11. Sharma SD, Budrukkar A, Upreti RR et al. Dosimetric comparison of conventional radiograph- and three-dimensional computed tomography-based planning using dose volume indices for partial breast intraoperative implants. Clin Oncol (R Coll Radiol) 2008; 20: 46-52.

12. Cuttino LW, Todor D, Arthur DW. CT-guided multi-catheter insertion technique for partial breast brachytherapy: reliable target coverage and dose homogeneity. Brachytherapy 2005; 4: 10-17.

13. Major T, Fröhlich G, Lövey K et al. Dosimetric experience with accelerated partial breast irradiation using image-guided interstitial brachytherapy. Radiother Oncol 2009; 90: 48-55.

14. Shah C, Wobb J, Manyam B et al. Accelerated partial breast irradiation utilizing brachytherapy: patient selection and workflow. J Contemp Brachytherapy 2016; 8: 90-94.

15. Gurram L, Wadasadawala T, Joshi K et al. Multi-catheter interstitial brachytherapy for partial breast irradiation: an audit of implant quality based on dosimetric evaluation comparing intra-operative versus post-operative placement. J Contemp Brachytherapy 2016; 8: 116-121.

16. Landis DM, Luo WL, Song J et al. Variability among breast radiation oncologists in delineation of the postsurgical lumpectomy cavity. Int J Radiat Oncol Biol Phys 2007; 67: 1299-1308.

17. Petersen RP, Truong PT, Kader HA et al. Target volume delineation for partial breast radiotherapy planning: clinical characteristics associated with low interobserver concordance. Int J Radiat Oncol Biol Phys 2007; 69: 41-48.

18. Kosztyla R, Olson R, Carolan H et al. Evaluation of dosimetric consequences of seroma contour variability in accelerated partial breast irradiation using a constructed representative seroma contour. Int J Radiat Oncol Biol Phys 2012; 84: 527-532.

19. Yang TJ, Tao R, Elkhuizen PHM et al. Tumor bed delineation for external beam accelerated partial breast irradiation: a systematic review. Radiother Oncol 2013; 108: 181-189.

20. Struikmans H, Wárlám-Rodenhuis C, Stam T et al. Interobserver variability of clinical target volume delineation of glandular breast tissue and of boost volume in tangential breast irradiation. Radiother Oncol 2005; 76: 293-299.

21. Major T, Gutiérrez C, Guix B et al. Interobserver variations of target volume delineation in multicatheter partial breast brachytherapy after open cavity surgery. Brachytherapy 2015; 14: 925-932.

22. Budrukkar A, Sarin R, Jalali R et al. Accelerated partial breast irradiation with interstitial brachytherapy as a sole modality of radiation for women with low risk early stage breast cancer. 2006 ASCO Annual Meeting Proceedings Part I. J Clin Oncol 2006; 24 (18S): 1066.

23. Kouwenhoven E, Giezen M, Henk Struikmans H. Measuring the similarity of target volume delineations independent of the number of observers. Phys Med Biol 2009; 54: 2863-2873.

24. Smitt MC, Birdwell RL, Goffinet DR. Breast electron boost planning: comparison of CT and US. Radiology 2001; 219: 203-206.
25. Major T, Gutiérrez C, Guix B et al. Recommendation from GEC ESTRO breast cancer working group (II): Target definition and target delineation for accelerated or boost partial breast irradiation using multicatheter interstitial brachytherapy after breast conserving open cavity surgery. Radiother Oncol 2016; 118: 199-204. 